\title{
Association Between Anxiety Level of Child with Parental and Patient Factors During Preoperative Anesthesia Visit ${ }^{\S}$
}

\author{
Rovnat Babazade $^{1,3}$, Burak Dogangun ${ }^{*}, 2$, Pervin Sutas-Bozkurt ${ }^{3}$, Gurcan Gungor ${ }^{3}$ and \\ Levent Kayaalp ${ }^{2}$
}

\author{
${ }^{I}$ Outcomes Research, Anesthesiology Institute, Cleveland Clinic, Cleveland, OH 44195, USA \\ ${ }^{2}$ Child and Adolescent Psychiatry, Istanbul University, Cerrahpasa Faculty of Medicine, Istanbul, Cerrahpasa, 34098, \\ Turkey \\ ${ }^{3}$ Anesthesiology and Reanimation, Istanbul University, Cerrahpasa Faculty of Medicine, Istanbul, Cerrahpasa, 34098, \\ Turkey
}

\begin{abstract}
Introduction: Preoperative anxiety in children is an important concern for the anesthesiologist. In our study, we aimed to determine the association between parental and patient factors with patients' anxiety during the preoperative anesthesia visit and additionally, aimed to inform clinicians about the parents' concerns and effects of culture.

Methods: Following the faculty ethical committee's approval, written consent was obtained from the parents of eligible children undergoing various surgical and radiological procedures under general anesthesia. The parents were given a socio-demographic questionnaire and both the child and the parents completed the Spielberger State -Trait Anxiety Inventory (STAI).

Results: A total of 117 children were enrolled. The majority of children were boys $(67 \%)$ and the mean age was 10.2 (years). Children had mean state anxiety scores of $47.8 \pm 7.4$ and mean trait anxiety scores of $33.8 \pm 7$. There was a significant negative correlation between child state and child trait anxiety $(\mathrm{r}=-.39$, moderate strength, $\mathrm{p}<0.001)$. Parent's state and trait anxiety scores had significant correlation. Poorly educated parents had significantly higher state anxiety scores $(p<0.001)$. We did not find any statistically significant relationship between other factors and state anxiety scores of either the children or the parents. Eleven of the parents who had replied to the open ended question $(n=55)$ asked for further information about the surgery and anesthesia to reduce anxiety level; others mainly stated that they were confident that the physicians will do their best to take care of their children.

Conclusion: Anxiety states are not universal. Conflicting relations between state and trait anxiety and socio-demographic factors affecting anxiety may differ in different countries and cultures.
\end{abstract}

Keywords: Anxiety, child, anesthesia, parents, education, social class.

\section{INTRODUCTION}

An estimated 6 million children undergo surgery and anesthesia each year in the United States alone, so the behavioral and clinical assessments of children and parents are an important concern for the anesthesiologist. Most parents and children awaiting the preoperative anesthesia visit and elective surgery experience preoperative anxiety and fear [1-3]. Anxiety may adversely influence anesthetic

*Address correspondence to this author at the Istanbul University, Cerrahpasa Faculty of Medicine, Child and Adolescent Psychiatry, Istanbul, Cerrahpasa, 34098, Turkey; Tel: +9 0532472 2822; Fax: +90 414 3128; E-mails: burakdogangun@hotmail.com, dogangun@istanbul.edu.tr

${ }^{\S}$ This study was presented by Rovnat Babazade as a poster in $66^{\text {th }}$ PostGraduate Assembly in Anesthesiology, New York, US, December, 2012

This study was registered to www.ClinicalTrials.gov under the NCT02201680 identifier

$\mathrm{http}: / /$ www.duplichecker.com was used for plagiarism check induction, patient recovery [4-6], and patient satisfaction, and may cause patients to avoid a planned operation [7]. Studies have shown that this anxiety is influenced by uncertainty of the forthcoming anesthetic and surgical procedures, past experiences, educational and socioeconomic status, patients' and parents' personality, ethnicity, culture, and coping style [8,9]. National and cultural diversity may also influence anxiety. However, in the last three decades clinicians have struggled to determine the sources of anxiety and to reduce preoperative anxiety in children, because studies are still insufficient in determining the associations of these known factors on anxiety during the preoperative anesthesia visit. Additionally, the literature addressing the relationship between pediatric patients' anxiety with parental and cultural factors are limited.

Thus, we aimed to design a study to test the association between parental parameters (anxiety, gender, and presence of psychiatric problems in the family, socio-economic, marital status, previous experience with surgery and chronic diseases, and education level) and patient factors (anxiety, 
age, gender, surgical risk, and previous operation) with patient's anxiety during the preoperative anesthesia visit. We also aimed to inform clinicians about the parents' concerns and effects of culture on anxiety.

\section{MATERIAL AND METHOD}

With ethical committee approval, we obtained written consent from the parents of literate, pediatric patients, aged 7 to 17 years, who applied for a preoperative anesthesia visit in a university hospital for various inpatient and outpatient surgical or radiological procedures requiring general anesthesia. Children with disruption of vision due to eye problems, and children with illiterate parents or parents diagnosed with any level of mental retardation were excluded. Written consent was obtained during the preoperative anesthesia visit, which is routinely performed one to seven days prior to the scheduled procedure.

One parent was asked to complete a 14-item questionnaire about their life, which included information about their level of education, employment status, total monthly income, other children in the family, family history of psychiatric problems, marital status, and previous experiences with surgery and chronic diseases. If both parents were accompanying the child, they decided amongst themselves which parent would be included. None of the children received drugs for anxiety before the preoperative visit. Additionally, the participating parent completed the 40question Spielberger State-Trait Anxiety Inventory (STAI), which measures state anxiety, or anxiety about an event, and trait anxiety, or anxiety as a personal characteristic (the Turkish translation of the STAI was validated for adults in 1998 by Oner, et al.) [10]. Oner, et al. reported that the state anxiety test and trait anxiety tests had reliability and validity values ranging from 0.83 to 0.92 and 0.86 to 0.92 , respectively [10]. The children were given the STAI with two separate lists of questions, each with 20 items (the Turkish translation was validated by Ozusta, et al. in 1995) [11]. It was stated that the Cronbach alpha value for children state and trait anxiety are 0.82 and 0.81 , respectively [11]. The scores were calculated as instructed by Ozusta [11]. These scores were categorized as no anxiety (scores 0-19), mild anxiety (scores 20-39), moderate anxiety (scores 4059), and severe anxiety (scores 60 and more) [10, 12].

No further contact was made with the child and parents as all children were anesthetized and operated on in different departments of the 3000-bed university hospital.

Based on knowledge from previous studies, 19-point changes in anxiety scores were reported as clinically significant $[10,12]$. The sample size calculation was estimated to be 117 patients for type I error of 0.05 with a statistical power of $80 \%$. Data were analyzed by SPSS 20 (Statistical Packet for Social Sciences). T-test for independent samples was used for comparison of factors containing two categories. One-way analysis of variance was used for comparisons of variables consisting of three and more categories and post hoc tests performed by Tukey range tests. Pearson correlation analysis was used to determine the relationship between parent and children state and trait anxiety scores. The definition of Dancey and Reidy was used in defining the strength of correlation $(\mathrm{r}=0.1-0.3$ weak, $0.3-0.6$ moderate strength correlation) [13]. Statistical significance level was accepted as $\mathrm{p}<0.05$.

\section{RESULTS}

A total of one-hundred-seventeen eligible children were enrolled in this study. The majority of children were boys $(67 \%)$ and the mean age of the patients was 10.2 (years). Nine percent of the children had examinations, radiological evaluation, or a minor surgery under general anesthesia (GA). Sixty-two percent underwent moderate surgeries $(n=16$ circumcision, $n=12$ tonsillectomy etc.). Twenty-nine percent of patients underwent major surgical intervention $(\mathrm{n}=5$ hypospadias repair, $\mathrm{n}=2$ vitrectomy, $\mathrm{n}=2$ upper pelvic juncture stricture, splenectomy, renal surgery etc.) (Table 1).

Children had an average state anxiety score of $47.8 \pm 7.4$ and an average trait anxiety score of $33.8 \pm 7$. The most frequent state anxiety was moderate anxiety level, whereas the most frequent trait anxiety was mild anxiety level (Table 2). There was a significant negative correlation between child state and child trait anxiety $(\mathrm{r}=-0.39$, moderate strength, $\mathrm{p}<0.001)$. Parental state anxiety $(\mathrm{r}=0.02, \mathrm{p}>0.05)$, parental trait anxiety $(\mathrm{r}=-0.08, \mathrm{p}>0.05)$, and child age $(\mathrm{r}=0.01, \mathrm{p}>0.05)$ were not significantly correlated with child state anxiety (Table 3 ). There was no significant change in child state anxiety according to the presence of parental psychiatric problems, previous operations in the family, chronic diseases in the family, previous operation of the child, marital status of parent(s), and working mothers $(p>0.05)$. In addition, the state anxiety of the child was not affected by the gender of the parent who completed the inventory $(\mathrm{p}>0.05)$ (Table 4). Child state anxiety scores were not affected by parental education, economical status of the family, or risk of the surgery (ANOVA, p>0.05) (Table 5).

Parents had an average anxiety score of $43.4 \pm 7.3$ and the highest incidence was moderate anxiety (Tables $\mathbf{1}$ and $\mathbf{2}$ ). Parents' state anxiety had a significant positive correlation with trait anxiety $(\mathrm{r}=0.44$, moderate strength, $\mathrm{p}<0.001)$. Child's age $(\mathrm{r}=-0.02, \mathrm{p}>0.05)$ was not significantly correlated with parental state anxiety.

Most of the parents were poorly educated; the ratio of parents that graduated from primary school (five years of primary education) and secondary school (three years after primary school) was $46 \%$ and $16 \%$ respectively. Poorly educated parents had significantly higher state anxiety scores $(\mathrm{p}<0.001)$ (Table 6).

The majority of parents (95\%) did not have any psychiatric problems (Table 1), however, presence of psychiatric problems in the family was a confounding factor on parent state anxiety ( $T$ test, $p<0.05$ ). Thirty-two percent of families had poor income level, although all of them had governmental insurance coverage for health care. Mother's working status, parents living together or not, previous operation of the child, and previous operation in the family or chronic disease of the family had no effect on trait anxiety of the parent ( $\mathrm{T}$ test, $\mathrm{p}>0.05$ ). Surgical risk and economic status of the family did not affect the parent state anxiety (ANOVA, $\mathrm{p}>0.05$ ). 
Table 1. Demographic characteristic and the anxiety scores.

\begin{tabular}{|c|c|c|c|c|}
\hline & $\mathbf{N}$ & $\%$ & Mean & Min-Max \\
\hline Child State Anxiety & & & $47.8 \pm 7.4$ & $27-60$ \\
\hline Child's Age (Year ) & & & $10.4 \pm 2.7$ & $7-17$ \\
\hline \multicolumn{5}{|l|}{ Child's Gender } \\
\hline Girl & 39 & 33 & & \\
\hline \multicolumn{5}{|l|}{ Surgical Risk } \\
\hline Examination under GA/Radiological Evaluation minor surgery & 11 & 9 & & \\
\hline Moderate surgical Intervention & 72 & 62 & & \\
\hline Yes & 54 & 46 & & \\
\hline Parent State Anxiety & & & $43.4 \pm 7.3$ & $25-66$ \\
\hline Parent Trait Anxiety & & & $48.2 \pm 6.9$ & $32-68$ \\
\hline \multicolumn{5}{|l|}{ Parent's Gender } \\
\hline Male & 50 & 43 & & \\
\hline Female & 67 & 57 & & \\
\hline \multicolumn{5}{|l|}{ Previous Operation /Chronic Disease in the Family } \\
\hline No & 43 & 37 & & \\
\hline University & 14 & 12 & & \\
\hline \multicolumn{5}{|l|}{ Economical Status } \\
\hline Poor & 38 & 32 & & \\
\hline Intermediate & 65 & 56 & & \\
\hline Good & 14 & 12 & & \\
\hline \multicolumn{5}{|l|}{ Parental Psychiatric Problem } \\
\hline Yes & 6 & 5 & & \\
\hline No & 111 & 95 & & \\
\hline \multicolumn{5}{|l|}{ Marital Status } \\
\hline Single Parent & 3 & 3 & & \\
\hline Married & 114 & 97 & & \\
\hline
\end{tabular}

Fifty-percent of parents replied to the open-ended question "what would you like to decrease your anxiety?" Eleven asked for further information about the surgery and anesthesia, while the others mainly stated that they are confident that the physicians will do their best to take care of their children.

\section{DISCUSSION}

Interestingly, we did not find a relationship between most of the expected parameters and anxiety level. Our results showed that there was a significant negative correlation at the moderate level between child state and child trait anxiety. 
Table 2. Anxiety level categorization, incidence of appearance.

\begin{tabular}{|c|c|c|c|c|c|c|c|c|}
\hline & \multicolumn{4}{|c|}{ Children } & \multicolumn{4}{|c|}{ Parent } \\
\hline & \multicolumn{2}{|c|}{ State Anxiety } & \multicolumn{2}{|c|}{ Trait Anxiety } & \multicolumn{2}{|c|}{ State Anxiety } & \multicolumn{2}{|c|}{ Trait Anxiety } \\
\hline & $\mathbf{N}$ & $\%$ & n & $\%$ & n & $\%$ & n & $\%$ \\
\hline No Anxiety (0-19) & 0 & 0 & 0 & 0 & 0 & 0 & 0 & 0 \\
\hline Mild (20-39) & 18 & 14.5 & 89 & 71.8 & 36 & 29 & 8 & 6.5 \\
\hline Moderate (40-59) & 96 & 77.4 & 28 & 22.6 & 78 & 62.9 & 104 & 83.9 \\
\hline High (60-79) & 3 & 2.4 & 0 & 0 & 3 & 2.4 & 5 & 4 \\
\hline
\end{tabular}

Table 3. Pearson correlation analysis of child state anxiety, parent state anxiety, parent trait anxiety.

\begin{tabular}{|c|c|c|c|c|c|c|}
\hline & \multicolumn{2}{|c|}{ Child Trait Anxiety } & \multicolumn{2}{c|}{ Parent State Anxiety } & \multicolumn{2}{c|}{ Parent Trait Anxiety } \\
\hline \hline \multirow{2}{*}{ Child State Anxiety } & $\mathrm{R}$ & $\mathrm{p}$ & $\mathrm{R}$ & $\mathrm{p}$ & $\mathrm{r}$ & $\mathrm{P}$ \\
\cline { 2 - 7 } & $\mathbf{- 0 . 3 9}$ & $\mathbf{0 . 0 0 0}$ & 0.02 & 0.806 & -0.08 & 0.395 \\
\hline
\end{tabular}

Table 4. Comparisons of child state anxiety scores according to demographic variables by test for independent sample analysis.

\begin{tabular}{|c|c|c|c|c|c|c|}
\hline Child State Anxiety & & $\mathbf{n}$ & Mean & Sd & $\mathbf{T}$ & $\mathbf{p}$ \\
\hline Parental Psychiatric Problem & $\begin{array}{l}\text { No } \\
\text { Yes }\end{array}$ & $\begin{array}{c}111 \\
6\end{array}$ & $\begin{array}{l}47.71 \\
49.50\end{array}$ & $\begin{array}{l}7.37 \\
9.35\end{array}$ & -0.571 & 0.569 \\
\hline Previous Operation in the Family/Chronic Disease of the Family & $\begin{array}{l}\text { No } \\
\text { Yes }\end{array}$ & $\begin{array}{l}43 \\
74\end{array}$ & $\begin{array}{l}48.23 \\
47.55\end{array}$ & $\begin{array}{l}7.64 \\
7.38\end{array}$ & 0.473 & 0.637 \\
\hline Mother's Working Status & $\begin{array}{l}\text { No } \\
\text { Yes }\end{array}$ & $\begin{array}{l}92 \\
25\end{array}$ & $\begin{array}{l}48.05 \\
46.88\end{array}$ & $\begin{array}{l}7.06 \\
8.84\end{array}$ & 0.697 & 0.487 \\
\hline
\end{tabular}

Hence, parents' state and trait anxiety levels presented a moderate correlation. Also poorly educated parents had significantly higher state anxiety scores $(p<.001)$. Stateanxiety is a transient emotional condition consisting of feelings of tension, apprehension, nervousness, and worriness. State anxiety varies in strength and changes over time as acute events in life. Trait-anxiety is individual and related to anxiety proneness, like a personality trait; it is stable over time [14]. High state-anxiety scores indicate high levels of anxiety at evaluation, such as the preoperative state in our study. High levels of trait-anxiety presents as an anxious personality trait.

Several authors previously reported that state-anxiety varied as a function of trait-anxiety [14-16]. Our results revealed significant negative correlation between the state and trait anxiety scores of children. In our pediatric group there was a high incidence of mild to moderate trait anxiety scores. This subclinical level of trait anxiety of children could have been exaggerated by psychological stressor factors due to surgery resulting in negative correlation (Table 2).

Similar to ours, a study from Turkey reported no relation between the families' preoperative state anxiety level and their children's anxiety upon arrival to the operating room, at induction, and at the $13^{\text {th }}$ minute in the recovery room [17]. Previous studies in the 1990s from the United States [18, 19] reported that a child's anxiety is strongly affected by the state- and trait-anxiety of the parent. Gil, et al. evaluated the trait-anxiety of parents of adolescents (12 -18 years old) in the postoperative period [18]. In another study Kain, et al. evaluated the trait anxiety of parents of 2- 10 years old children [19]. This discrepancy with previous literature can be related to cultural differences between countries, as cultural factors have been shown to influence level of anxiety [9]. In Turkey, the family structure is quite different from European and American societies in that families have an extended family structure. Grandparents, close relatives, and even friends may act as a barrier in reflection of parent's anxiety to the child.

Caumo, et al.'s study of 90 school children in Brazil, reported that children have trait-anxiety scores of $38.58 \pm$ 6.54 , which is higher than the scores found in our study group, whereas the state-anxiety scores in the preoperative period was $32.12 \pm 7.95$ which is lower than our group. In Caumo, et al.'s study, the people accompanying the children had similar trait anxiety scores $(43.04 \pm 11.31)$ and of the 
Table 5. Comparisons of child state anxiety results according to parental education, economical status and surgical risk by one way analysis of variance (ANOVA).

\begin{tabular}{|c|c|c|c|c|c|}
\hline \multicolumn{2}{|r|}{ Child State Anxiety } & $\mathbf{N}$ & Mean & SD & $\mathbf{P}$ \\
\hline \multirow{4}{*}{ Parental Education } & Primary School & 54 & 46.02 & 7.66 & \multirow{4}{*}{0.076} \\
\hline & Secondary School & 19 & 48.79 & 6.46 & \\
\hline & High School & 30 & 51.14 & 7.14 & \\
\hline & University & 14 & 47.80 & 7.45 & \\
\hline \multirow{3}{*}{ Economical Status } & Poor & 38 & 47.34 & 7.21 & \multirow{3}{*}{0.828} \\
\hline & Intermediate & 65 & 48.18 & 7.11 & \\
\hline & Good & 14 & 47.29 & 9.81 & \\
\hline \multirow{3}{*}{ Surgical Risk } & Physical Examination under General Anesthesia & 11 & 49.09 & 7.54 & \multirow{3}{*}{0.778} \\
\hline & Minor Surgical Intervention & 72 & 47.86 & 8.12 & \\
\hline & Major Surgical Intervention & 34 & 47.26 & 5.92 & \\
\hline
\end{tabular}

state-anxiety scores $(44.76 \pm 9.76)$ to ours. In Brazilian children low level of trait-anxiety was reported; around $50 \%$ of the children and $57 \%$ of the parents. Our results were quite different from their incidences of high and low levels of anxiety. The most important issue is the definition of high and low levels; the citation in this literature was in Portuguese, so we are unable to get this information (Table 3). In comparison to our results they report that there was no significant association between the degree of postoperative state-anxiety of the children and sex, age, years in school, history of chronic disease or previous surgery, length of anesthesia and time to recovery, dose of midazolam, trait anxiety of the child, and state- and trait-anxiety of the person responsible for her/him.

Table 6. Comparisons of parent state anxiety results according to parental education by one way analysis of variance (ANOVA).

\begin{tabular}{|c|c|c|c|c|c|c|}
\hline \multicolumn{2}{|c|}{ Parent State Anxiety } & $\mathbf{N}$ & Mean & Sd & $\mathbf{P}$ & Difference \\
\hline \multirow{4}{*}{$\begin{array}{c}\text { Parental } \\
\text { Education }\end{array}$} & $\begin{array}{l}\text { Primary } \\
\text { School }\end{array}$ & 54 & 46.37 & 7.72 & \multirow{4}{*}{.000} & $\begin{array}{l}1>2 \\
1>3\end{array}$ \\
\hline & $\begin{array}{c}\text { Secondary } \\
\text { School }\end{array}$ & 19 & 39.95 & 3.63 & & \\
\hline & $\begin{array}{l}\text { High } \\
\text { School }\end{array}$ & 30 & 41.07 & 6.93 & & \\
\hline & University & 14 & 42.00 & 5.99 & & \\
\hline
\end{tabular}

Several authors had emphasized previous hospitalization and complications increase children's anxiety responses [16, 19]. Our study revealed no relationship between previous surgical experience and level of preoperative anxiety. Different cultures and nations have different perceptions of operations and coping skills of anxiety and develop different cultural defense mechanisms. In our previous study, performed in pediatric patients at the same hospital, we found that repeated anesthesia did not seem to disturb the child's psychological health further when tentative and precautious approach modalities were undertaken. In this study, we were not aware of the conditions of anesthesia and surgery techniques performed in previous operations because many had been treated at other hospitals.

In the literature, there are several studies which have examined risk factors for heightened anxiety prior to anesthesia in children. Davidson, et al. reported that factors most clearly associated with high anxiety were younger age, anxious parents at induction, previous behavioral problems at healthcare attendances, having a procedure greater than 30 minutes in duration and being admitted to a hospital more than five times previously [16]. Kain, et al. [19] reported in a younger study population than ours (1-5 years old) that younger children had the highest risk for developing extreme anxiety. In our study, age ranged between 7-17 years old and we did not find any significant association between anxiety level and age. While this could be due to the ages, another explanation may be that our older patient population were less uncertain and fearful of the anesthesia and surgery when compared to 1-5 year old children.

We found a positive relationship between state-anxiety and trait-anxiety levels of the patients' parents. Our study indicates a negative correlation between parents' educational status and anxiety level. As the families' education level increases, coping skills related to surgery may increase the capacity of problem solving. In addition, further education including university degrees, knowledge about surgery, and understanding of anesthesia complications may reduce anxiety.

Ten percent of parents (only $50 \%$ replied to the open-ended question) have commented that receiving further information about the surgery and anesthesia would decrease their anxiety; most of these parents had university degrees. Fortier, et al., pointed out that not only parents, but also children are seeking out information in attempts to manage their anxiety [20]. Kain, et al. had pointed out in 1997 that the vast majority of parents prefer to have comprehensive information concerning their child's general anesthetic, including information about all possible complications [21]. Moreover, in parents of children undergoing general anesthesia and outpatient surgery, very detailed anesthetic information of what might go wrong does not increase parental anxiety, and has the advantage of allowing parents a fully informed choice. 
A limitation of our study is that the patients enrolled were covered by a governmental insurance company, so we missed approximately $5 \%$ of the population covered by private insurance companies (wealthy population). However, patients in Istanbul, and especially university hospital patients, are the best representation of the whole Turkish population and cultural wealth, which is an advantage that made our study stronger. Another limitation of our study is that we evaluated the anxiety level of the child during the preoperative anesthesia visit. Patient's anxiety level may differ on the day of the preoperative visit than on the day of surgery or after the surgery.

In summary, we expected to find a significant relationship between anxiety level and all parental parameters and patient factors in our study during the preoperative anesthesia visit; our results did not support those relationships. In our opinion, anxiety states are not universal and conflicting relations between state and trait anxiety and socio- demographic factors affecting anxiety may differ in different countries and cultures. Further cultural- and country-based studies are required to guide and inform clinicians about how to approach the patients from different cultures.

\section{AUTHORS CONTRIBUTION}

R.B.: concept and design and the predictors selection; conduct of study, data analysis and interpretation of data, drafting the article or revising it critically for important intellectual content

B.D.: concept and design, and the predictors selection, conduct of study; drafting the article or revising it critically for important intellectual content; reviewed data analysis

P.S.B.: data analysis and interpretation of data; drafting the article or revising it critically for important intellectual content, and the predictors selection

G.G.: data analysis, drafting the article or revising it critically for important intellectual content

L. K.: interpretation of data; drafting the article or revising it critically for important intellectual content

\section{CONFLICT OF INTEREST}

The authors confirm that this article content has no conflict of interest.

\section{ACKNOWLEDGEMENTS}

The study was approved by the Faculty Ethical Committee of the Istanbul University, Cerrahpasa Medical Faculty. The authors have no conflict of interest to declare. The authors would like to thank Erdal Yanardoner who contributed to statistical and data analysis, and Matthew T. Hutcherson who contributed to the preparation of the manuscript.

\section{REFERENCES}

[1] Badner NH, Nielson WR, Munk S, et al. Preoperative anxiety: detection and contributing factors. Can J Anaesth 1990; 37: 444 -7.

[2] Domar AD, Everett LL, Keller MG. Preoperative anxiety: is it a predictable entity? Anesth Analg 1989; 69: 763-7

[3] Johnston M. Anxiety in surgical patients. Psychol Med 1980; 10: 14552 .

[4] Scott LE, Clum GA, Peoples JB. Preoperative predictors of postoperative pain. Pain 1983; 15: 283-93.

[5] Quinn AC, Brown JH, Wallace PG, et al. Studies in postoperative sequelae: nausea and vomiting-still a problem. Anaesthesia 1994; 49: $62-5$.

[6] Goldmann L, Ogg TW, Levey AB. Hypnosis and daycase anaesthesia: a study to reduce pre-operative anxiety and intraoperative anaesthetic requirements. Anaesthesia 1988; 43: 466-9.

[7] McCleane GJ, Cooper R. The nature of pre-operative anxiety. Anaesthesia 1990; 45: 153-5.

[8] Martin SR, Fortier MA, Kain DI, et al. Desire for perioperative information and parental ethnicity. Paediatr Anaesth 2011; 21: 104651

[9] Shirley PJ, Thompson N, Kenward M, et al. Parental anxiety before elective surgery in children A British perspective. Anaesthesia 1998; 53: 956-9

[10] Öner, N, Le Compte, A. Durumluk-Sürekli Kaygı Envanteri El Kitabı [State-Trait Anixety Inventory Handbook]. İstanbul: Boğaziçi Üniversitesi Yayınları 1998.

[11] Ozusta S. Çocuklar için durumluk-sürekli kaygı envanterinin uyarlama, geçerlik ve güvenirlik çalışması. [Application of state-trait anxiety inventory for children validity and reliaabiility]Türk Psikoloji 1995; 10(32): 44.

[12] Turan NK, Acaroglu R. The relationship between anxiety levels of adolescents who undergo surgical interventions and their parents and analysis of anxiety causes. Turkiye Klinikleri J Med Sci 2012; 32: 308-15

[13] Dancey C, Reidy J. Statistics without Maths for Psychology. Published by Prentice Hall 2004.

[14] Caumo W, Broenstrub JC, Fialho L, et al. Risk factors for postoperative anxiety in children. Acta Aanaesth Scan 2000; 44: 782-9

[15] Chapman CR, Cox GB. Anxiety, pain, and depression surrounding elective surgery: a multivariate comparison of abdominal surgery patients with kidney donors and recipients. J Psychosom Res 1977; 21: 7-15

[16] Davidson AJ, Shrivastava PP, Jamsen K, et al. Risk factors for anxiety at induction of anesthesia in children: a prospective cohort study. Pediatr Anesth 2006; 16(9): 919-27.

[17] Bal N, Saricaoglu F, Uzun S, et al. Perioperative anxiety and postoperative behavioural disturbances in children: comparison between induction techniques. Eur J Anaesth 2006; 23(6): 470-5.

[18] Gil KM, Ginsberg B, Muir M, et al. Patient controlled analgesia: the relation of psychological factors to pain and analgesic use in adolescents with postoperative pain. Clin J Pain 1992; 8(3): 215-21

[19] Kain, ZN, Mayes LC, O'Connor TZ, et al. Preoperative anxiety in children: predictors and outcomes. Arch Ped Adol Med 1996; 150: 1238-45.

[20] Fortier MA, Chorney JM, Rony R, et al. Children's desire for perioperative information. Anesth Analg 2009; 109(4): 1085

[21] Kain ZN, Wang SM, Caramico LA, et al. Parental desire for perioperative information and informed consent: a two-phase study. Anesth Analg 1997; 84(2): 299-306. 